\title{
Optimizing de novo common wheat transcriptome assembly using short-read RNA-Seq data
}

\author{
Jialei Duan ${ }^{1,2}$, Chuan Xia ${ }^{1}$, Guangyao Zhao ${ }^{1}$, Jizeng Jia ${ }^{1 *}$ and Xiuying Kong ${ }^{1 *}$
}

\begin{abstract}
Background: Rapid advances in next-generation sequencing methods have provided new opportunities for transcriptome sequencing (RNA-Seq). The unprecedented sequencing depth provided by RNA-Seq makes it a powerful and cost-efficient method for transcriptome study, and it has been widely used in model organisms and non-model organisms to identify and quantify RNA. For non-model organisms lacking well-defined genomes, de novo assembly is typically required for downstream RNA-Seq analyses, including SNP discovery and identification of genes differentially expressed by phenotypes. Although RNA-Seq has been successfully used to sequence many non-model organisms, the results of de novo assembly from short reads can still be improved by using recent bioinformatic developments.

Results: In this study, we used 212.6 million pair-end reads, which accounted for $16.2 \mathrm{~Gb}$, to assemble the hexaploid wheat transcriptome. Two state-of-the-art assemblers, Trinity and Trans-ABySS, which use the single and multiple k-mer methods, respectively, were used, and the whole de novo assembly process was divided into the following four steps: pre-assembly, merging different samples, removal of redundancy and scaffolding. We documented every detail of these steps and how these steps influenced assembly performance to gain insight into transcriptome assembly from short reads. After optimization, the assembled transcripts were comparable to Sangerderived ESTs in terms of both continuity and accuracy. We also provided considerable new wheat transcript data to the community.

Conclusions: It is feasible to assemble the hexaploid wheat transcriptome from short reads. Special attention should be paid to dealing with multiple samples to balance the spectrum of expression levels and redundancy. To obtain an accurate overview of RNA profiling, removal of redundancy may be crucial in de novo assembly.
\end{abstract}

\section{Background}

The advent of next-generation sequencing technology in recent years has provided new opportunities to the field of RNA sequencing (RNA-Seq), which has emerged as a powerful tool for transcriptome study. With advances in sequencing technology, RNA-Seq, which is cost-efficient and yields sufficient information, has been widely used in both well-studied model organisms and non-model organisms in various studies, including transcript profiling, SNP discovery, and the identification of genes that are differentially expressed between samples [1-5].

\footnotetext{
* Correspondence: jzjia@mail.caas.net.cn; xykong@mail.caas.net.cn 'Key Laboratory of Crop Gene Resources and Germplasm Enhancement, Ministry of Agriculture, National Key Facility for Crop Gene Resources and Genetic Improvement, Institute of Crop Science, Chinese Academy of Agricultural Sciences, No. 12 South Street, Zhongguancun, Beijing 100081, People's Republic of China

Full list of author information is available at the end of the article
}

For organisms with known reference genomes, mapping-first approaches have often been used for RNA-Seq analysis. Reads were first mapped to the annotated references. Then, the assembly of transcripts and the quantification of transcript expression levels were based on the mapping information. Alternatively, for those organisms lacking well-defined genomic references, these studies were typically performed using references to related species [6,7], assembled ESTs from target species $[5,8]$ or de novo assembly of RNA-Seq data [9]. To use sequences from related species as references, there must be a well-studied and closely related species. Moreover, mapping reads to a related organism may result in a loss of information regarding species-specific genes, and, additionally, no overview of the target transcriptome can be generated. Assembling ESTs from the organism of interest to serve as a reference requires the

\section{Biomed Central}


existence of comprehensive EST information, and researchers may lose tissue-specific information. This loss poses a problem because the reliability of the results partly depends on the quality of EST assembly. To gain an accurate overview of the transcriptome, de novo assembly is crucial for downstream RNA-Seq analyses. However, de novo assembly of the transcriptome has some unique challenges, particularly in the case of plants, which possess a large amount of paralogs and isoforms. Assembling non-normalized transcriptomes is different from assembling normalized transcriptomes and genomes because the read depth of transcripts is uneven, which, in turn, reflects differences in expression levels. Many de novo assembly projects for non-model organisms have used Roche 454 pyrosequencing technology (currently about $500 \mathrm{bp}$ ) because the length of reads generated are much longer than the short reads ( $<150$ bp currently) generated by Illumina SOLEXA and ABI SOLiD technologies. However, short-read technologies are much more economical. Recently, Oases, Trinity, and Trans-ABySS have been developed specifically for RNA-Seq assembly using short sequence reads and have been applied successfully in many experiments [10-12].

Common wheat (Triticum aestivum L., $2 n=6 x=42$ ) is one of the most important food crops in the world. The large genome $(16 \mathrm{~Gb})$, high proportion of repetitive sequences $(>80 \%)$, and hexaploid nature make complete sequencing of the wheat genome a daunting task for even state-of-the-art technologies. In addition to the identification of differentially expressed genes among different phenotypes to understand biological processes, due to the lack of high quality genomic sequence, RNA profiling is a practical approach to surveying the wheat genome. However, few wheat studies had taken advantage of RNA-Seq. Cantu et al. [13] assembled 1.4 million 454 reads into 30,497 contigs to study grain protein content gene in wheat. Li et al. [8] performed an mRNA tag analysis of wheat seedlings, rather than using assembly and mapped sequence reads to assemble public ESTs, to gain an overview of how wheat respond to $\mathrm{H}_{2} \mathrm{O}_{2}$ treatments. Pont et al. [7] assembled 934,928 reads generated from 454 sequences of normalized cDNA libraries into 37,695 sequence clusters, accounting for $20.1 \mathrm{Mb}$, to understand the polyploidization events of common wheat. Pellny et al. [6] utilized rice sequences and 1.5 million public ESTs as references to study the transcriptome of the developing starchy endosperm of common wheat. Trick et al. [5] mapped short reads from two samples to 40,349 unigene sequences (31.7 Mb) to identify SNPs. However, none of these groups performed de novo short read assembly from non-normalized wheat cDNA libraries.

Although RNA-Seq has been successfully used in many non-model organisms to understand transcriptome architecture and various biological processes, the results of de novo assembly from short reads still can be improved by using recent advances in bioinformatics, such as the method of merging single k-mer assemblies $[10,12,14]$. Strategies for dealing with multiple samples are an important aspect of de novo assembly that still lacks thorough discussion. In this study, we optimized de novo assembly of the hexaploid wheat transcriptome using 16.2 Gb short reads, and we documented every detail of the assembly process and discussed the effects of each assembly step to gain insight into the transcriptome assembly of non-model organisms. This study also provided comprehensive new transcript data to the wheat community.

\section{Results}

\section{Illumina sequencing}

To obtain a general overview of the common wheat transcriptome and an initial comparison of fertile and sterile wheat transcripts, four libraries (AF, AS, SF, SS) were constructed for paired end (PE) sequencing. AF, AS, SF, and SS were mix samples of fertile anthers, sterile anthers, fertile young spikes, and sterile young spikes of common wheat, respectively. A total number of 220.5 million $90 \mathrm{bp}$ PE reads accounting for $20.1 \mathrm{~Gb}$ of raw data were generated for the four libraries. After trimming the low-quality part at the 3' end of each read and filtering out singleton reads and reads less than $25 \mathrm{bp}$ in length, 212.6 million PE reads $(16.2 \mathrm{~Gb})$ were used for downstream analyses (Table 1). The GC content of raw reads dropped from about $51 \%$ to about $40 \%$, suggesting that our data may not have had good coverage across parts of the wheat transcriptome with high GC content (Table 1).

Table 1 Statistics of trimmed reads

\begin{tabular}{lllll}
\hline Libraries & Total reads & Total nucleotides (nt) & Average length & GC percentage \\
\hline AF & $33,067,246(1.5 \%)$ & $2,426,965,166(23.3 \%)$ & 73.4 & $39.15 \%(52.7 \%)$ \\
AS & $80,879,822(4.6 \%)$ & $6,204,420,998(18.7 \%)$ & 76.7 & $40.03 \%(50.8 \%)$ \\
SF & $32,678,050(1.3 \%)$ & $2,410,831,266(22.8 \%)$ & 73.8 & $38.95 \%(51.9 \%)$ \\
SS & $65,967,360(4.5 \%)$ & $5,148,749,102(17.1 \%)$ & 78.0 & $40.48 \%(50.3 \%)$ \\
\hline
\end{tabular}

The numbers in the parentheses indicated the trimmed proportions of reads, bases and the GC content before trimming. 


\section{Different strategies of de novo assembly}

The greatest concern for plant transcriptome de novo assembly is the misassembly of a large amount of paralogs and diverse alleles in plants. Considering the fact that common wheat has three sub-genomes, to gain the optimal assembly, several assembly strategies were used and their performance in assembling the wheat transcriptome was compared. We chose two state-of-the-art de Bruijn graph assemblers, Trinity [11] and Trans-ABySS [12], which use single $\mathrm{k}$-mer and multiple k-mer methods to generate assemblies, respectively. Trinity is reported to be efficient in recovering full-length transcripts and spliced isoforms [11], whereas Trans-ABySS is reported to yield optimal overall assembly covering wide transcript expression levels by merging multiple individual k-mer assemblies [12]. Moreover, because we had four libraries reflecting different phenotypes and tissues, we evaluated the performance of assembling each library separately and then merged them or assembled all libraries at the initial stage. For each of these two programs, we performed seven assemblies that can be divided into the following three categories: assembly of all four samples as a whole (designated as AFSFASSS), assembly of samples with the same phenotype (designated as AFSF and ASSS) and assembly of each of the four samples individually (designated as AF, SF, AS, and SS). AFSF and ASSS were then merged to form the final assembly (designated as AFSF + ASSS), as well as the four individual assemblies of AF, SF, AS, and SS (designated as $\mathrm{AF}+\mathrm{SF}+\mathrm{AS}+\mathrm{SS})$.

\section{Statistics of pre-assemblies}

For each of the Trans-ABySS assemblies, we used k-mer lengths from every odd number starting from 45 to 87 . To facilitate the subsequent merging step, the scaffolding option, which introduces Ns into the assembly results, was turned off. For every $\mathrm{k}$-mer length, the more reads that were used, the larger were the N50 and the total sum of assembly (Figure 1A, 1B). The largest N50 of individual kmer assemblies for AFSFASSS, AFSF, ASSS, AF, AS, SF, and SS was 623 bp (k-mer length of 61), 584 bp (53), 613 bp (63), 524 bp (53), 588 bp (55), $534 \mathrm{bp}$ (53), and $560 \mathrm{bp}$ (59), respectively. After merging individual $\mathrm{k}$-mer assemblies, the N50 slightly increased for all seven assemblies (Table 2). In contrast to the Trans-ABySS results, Trinity assemblies had much larger N50 and more contigs that were longer than $1 \mathrm{~kb}$. Alternatively, TransABySS produced much larger transcriptomes, which were on average 1.7 times larger than the results when using Trinity (Table 2).

\section{Merging of individual sample assemblies, redundancy reduction and scaffolding}

Assemblies from individual samples were merged using cd-hit-est with $100 \%$ identity [15] to form the overall assemblies. Contigs representing non-sample-specific genes existing across multiple samples were merged into single contigs. After merging individual samples, both the number and the total summed length of contigs had been reduced. The most redundant assembly was TransABySS_AF + SF + AS + SS, because the number of contigs had been reduced by $22.54 \%$. Assemblies produced by Trans-ABySS contained many more non-sample-specific contigs. The merged proportion of the four-samplemerging approach was higher than that of merging two assemblies (AFSF, ASSS, Table 3, Additional file 1: Figure S1, detailed statistics can be found in Additional file 2: Table S1). After merging separate sample assemblies, we had the following six pre-assemblies: three assemblies from Trinity and three from Trans-ABySS.

Contigs that overlapped with a minimum length of $50 \mathrm{bp}$ and minimum identity of $99 \%$ were collapsed into single contigs using GICL to further remove redundancy [16]. After this step, a large proportion of contigs had been merged (Figure 2, Table 3). Before deduplication (clustering related contigs to remove redundancy), assemblies produced by Trans-ABySS had a much greater number of contigs and total summed length than those of the counterpart Trinity assemblies. After the removal of redundancy, both the number of contigs and the summed lengths were lower than those of the counterpart Trinity assemblies (Table 3). Moreover, this deduplication step significantly increased the N50 of the three Trans-ABySS assemblies $(p=0.003)$. Merging individual assemblies, including multiple samples or multiple kmer assemblies, introduced more redundancy.

To reduce fragmental degree, contigs were joined using read pair information as implemented by SSPACE [17]. After scaffolding, the N50 of all six assemblies had been slightly increased by approximately $2.5 \%$ (Additional file 1: Figure S1, Table 3, Figure 3).

\section{Assessment of novelty and redundancy}

To determine whether the different assembly sizes and contig numbers were mainly due to novel sequences in each assembly or redundancy, pairwise comparisons of the six assemblies were performed using BLAT [18]. The novelty and redundancy of each assembly were determined by the proportion of base coverage by the other five assemblies with a high threshold (a minimum overlap length of $50 \mathrm{bp}$ and minimum identity of 99\%). Pairwise comparisons showed that the Trinity_AF + SF + AS + SS assembly had the most novel bases, whereas the TransABySS_AFSF + ASSS assembly had the fewest. Trinity assemblies had more novel sequences (on average, 79.15\% 

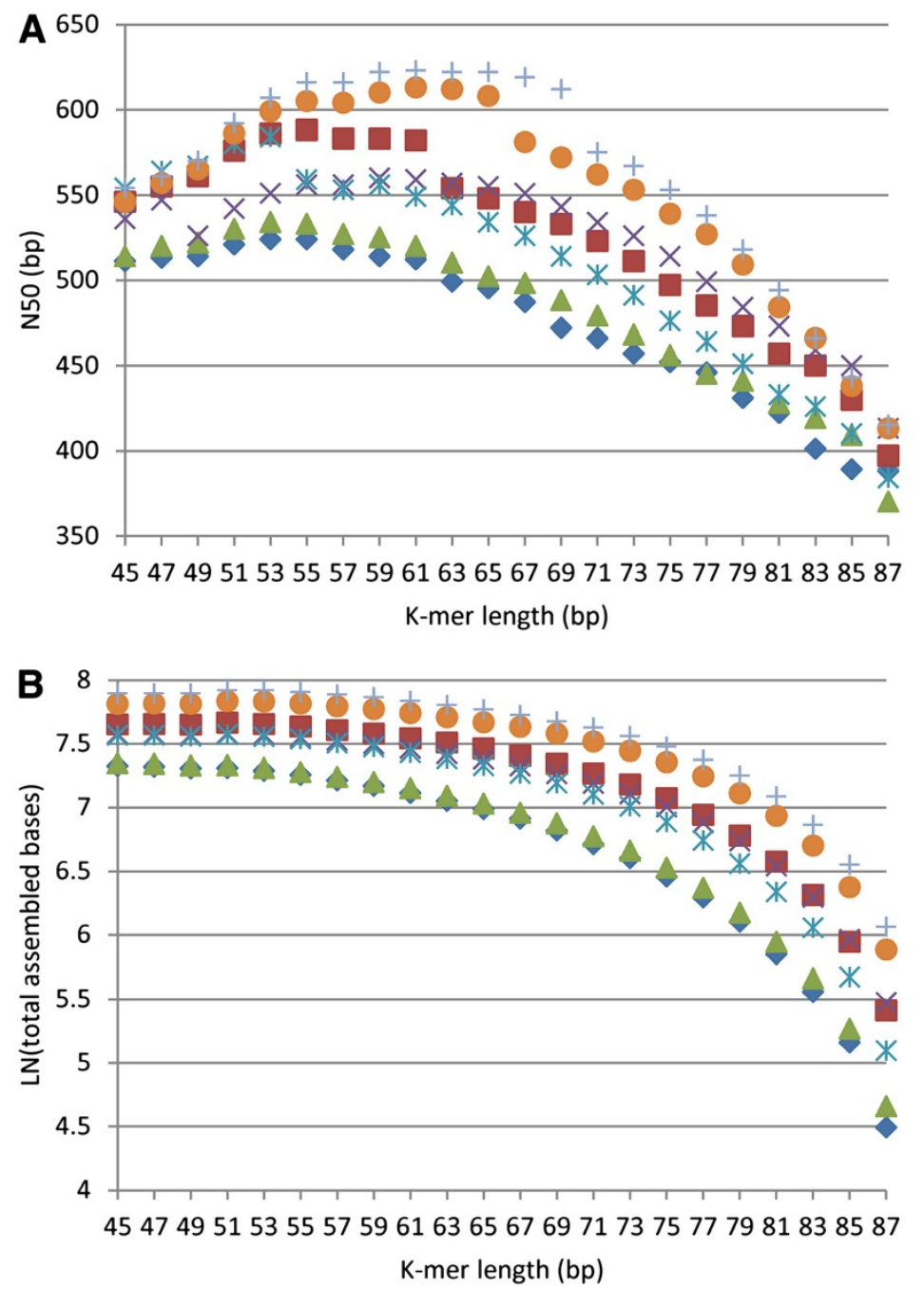

$\rightarrow \mathrm{N} 50: \mathrm{AF}$

N50:AS

N50:SF

$\times$ N50:SS

* N50:AFSF

N50:ASSS

+ N50:AFSFASSS

$\diamond$ sum:AF

sum:AS

sum:SF

$\times$ sum:SS

* sum:AFSF

sum:ASSS

+ sum:AFSFASSS

Figure $1 \mathrm{~N} 50$ and total length of the assemblies produced by ABySS with different k-mers. Every odd number starting from 45 to 87 were used as k-mer sizes, and different assembly strategies were compared. For example, AF refers to the use of reads from the AF sample for assembly, whereas AFSFASSS refers to the use of reads from all four samples together. A) N50, B) total length. The total length of each assembly is shown on a logarithmic scale.

of bases were covered) than those of Trans-ABySS (85.21\%, Figure 4, Additional file 3: Table S2). Assemblies produced by the same assembler were covered more frequently by each other (Figure 4, Additional file 3: Table S2).

To evaluate assembly redundancy, reads from all four samples were mapped to the six assemblies. The proportion of reads mapped to more than three locations was used as an indicator of redundancy. On average, after removing redundancy and scaffolding, Trans-ABySS assemblies were less redundant than Trinity assemblies (Table 4). Similarly, the merging samples approach resulted in more redundancy, whether using Trinity or Trans-ABySS. The most redundant assembly was Trinity_AF $+\mathrm{SF}+\mathrm{AS}+\mathrm{SS}$, of which $10.56 \%$ of its mapped reads had more than three hits. The contig number and total size of Trinity_AF + SF + AS + SS were also the largest among the six assemblies.

\section{Assessment of sample specificity}

To evaluate the performance of assembling all libraries at the initial stage and assembling each library separately and then merging them for each of the two assemblers, we performed three assemblies, AFSFASSS, AFSF + ASSS, $\mathrm{AF}+\mathrm{SF}+\mathrm{AS}+\mathrm{SS}$, representing the assembly of all samples, samples with the same phenotype, and individual samples at the initial stage, respectively. The purpose of this evaluation was to determine whether AFSFASSS and AFSF + ASSS lost a considerable amount of sample-specific transcripts or if AFSF + ASSS and $\mathrm{AF}+\mathrm{SF}+\mathrm{AS}+\mathrm{SS}$ contained more redundant transcripts and more fragmental transcripts. 
Table 2 Statistics of initial assembly

\begin{tabular}{|c|c|c|c|c|c|c|c|}
\hline & AFSFASSS & AFSF & ASSS & AF & AS & SF & SS \\
\hline \multicolumn{8}{|l|}{ Trinity } \\
\hline No. of contigs & 227,879 & 110,162 & 198,382 & 69,721 & 133,556 & 76,474 & 126,848 \\
\hline Total Bases & $217,485,766$ & $89,132,635$ & $184,623,552$ & $3,9925,904$ & $115,757,220$ & $55,932,501$ & $100,283,444$ \\
\hline No. of contigs (> = 1 kbp) & 69,402 & 28,016 & 58,759 & 6,817 & 36,825 & 16,409 & 30,694 \\
\hline Total Bases (in contigs $>=1$ kbp) & $135,112,080$ & $47,002,017$ & $112,433,788$ & $9,818,210$ & $65,495,871$ & $25,146,108$ & $50,760,639$ \\
\hline Max contig length & 12,488 & 7,710 & 10,506 & 5,723 & 11,199 & 5,670 & 5,294 \\
\hline Mean contig length & 954 & 809 & 930 & 572 & 866 & 731 & 790 \\
\hline N50 & 1,370 & 1,060 & 1,323 & 600 & 1,165 & 903 & 1,015 \\
\hline No. of contigs in N50 & 46,815 & 25,597 & 41,204 & 20,241 & 29,723 & 19,351 & 30,035 \\
\hline \multicolumn{8}{|l|}{ ABySS } \\
\hline No. of contigs & 571,835 & 258,087 & 478,056 & 157,039 & 334,220 & 158,856 & 279,549 \\
\hline Total Bases & $35,941,1943$ & $152,450,028$ & $296,430,486$ & $88,785,112$ & $202,564,854$ & $90,257,267$ & $165,879,838$ \\
\hline No. of contigs (> = 1 kbp) & 66,393 & 22,844 & 52,783 & 11276 & 33,779 & 11,757 & 25,615 \\
\hline Total Bases (in contigs > = 1 kbp) & $88,342,878$ & $29,539,332$ & $69,895,282$ & $14,500,455$ & $44,327,763$ & $14,876,118$ & $33,225,449$ \\
\hline Max contig length & 8,216 & 4,077 & 5,694 & 5,031 & 6,687 & 4,725 & 4,804 \\
\hline Mean contig length & 628 & 590 & 620 & 565 & 606 & 568 & 593 \\
\hline N50 & 686 & 634 & 674 & 597 & 653 & 604 & 637 \\
\hline No. of contigs in N50 & 178,083 & 82,822 & 149,467 & 51,187 & 105,491 & 51,861 & 89,188 \\
\hline
\end{tabular}

Contigs less than 300 bp were excluded.

Table 3 Statistics of each assembly step

\begin{tabular}{|c|c|c|c|c|c|c|}
\hline & \multicolumn{2}{|c|}{ AFSFASSS } & \multicolumn{2}{|c|}{ AFSF + ASSS } & \multicolumn{2}{|c|}{$A F+S F+A S+S S$} \\
\hline & Trinity & Trans-ABySS & Trinity & Trans-ABySS & Trinity & Trans-ABySS \\
\hline \multicolumn{7}{|l|}{ After merging $^{a}$} \\
\hline No. of contigs & 227,879 & 571,835 & 297,319 & 636,921 & 379,837 & 720,131 \\
\hline Total Bases & $217,485,766$ & $359,411,943$ & $267,708,300$ & $398,478,666$ & $298,801,419$ & $444,024,673$ \\
\hline N50 & 1,370 & 686 & 1,243 & 683 & 1,000 & 670 \\
\hline No. of contigs in N50 & 46,815 & 178,083 & 64,054 & 199,771 & 89,338 & 228,145 \\
\hline \multicolumn{7}{|l|}{ After deduplication } \\
\hline No. of contigs & 165,174 & 152,963 & 179,876 & 138,487 & 191,858 & 128,990 \\
\hline Total Bases & $139,479,895$ & $117,699,005$ & $151,369,559$ & $104,950,724$ & $151,376,565$ & $94,829,611$ \\
\hline N50 & 1,146 & 935 & 1,134 & 914 & 1,016 & 878 \\
\hline No. of contigs in N50 & 34,500 & 39,253 & 38,937 & 35,969 & 44,201 & 33,877 \\
\hline \multicolumn{7}{|l|}{ After scaffolding } \\
\hline No. of contigs & 162,090 & 152,694 & 176,983 & 138,452 & 188,653 & 129,464 \\
\hline Total Bases & $139,459,722$ & $120,117,056$ & $151,354,485$ & $107,614,336$ & $151,363,021$ & $97,866,545$ \\
\hline N50 & 1,177 & 960 & 1,161 & 942 & 1,041 & 907 \\
\hline No. of contigs in N50 & 33,941 & 39,124 & 38,409 & 35,915 & 43,481 & 33,927 \\
\hline Proportion of Ns & $0.12 \%$ & $0.13 \%$ & $0.12 \%$ & $0.13 \%$ & $0.12 \%$ & $0.13 \%$ \\
\hline GC content & $47.93 \%$ & $48.69 \%$ & $48.18 \%$ & $48.76 \%$ & $48.51 \%$ & $48.81 \%$ \\
\hline
\end{tabular}

a Trinity_AFSFASSS and Trans-ABySS_AFSFASSS had not been merged.

Detail statistics can be found in Additional file 2. Contigs less than 300 bp were excluded. 


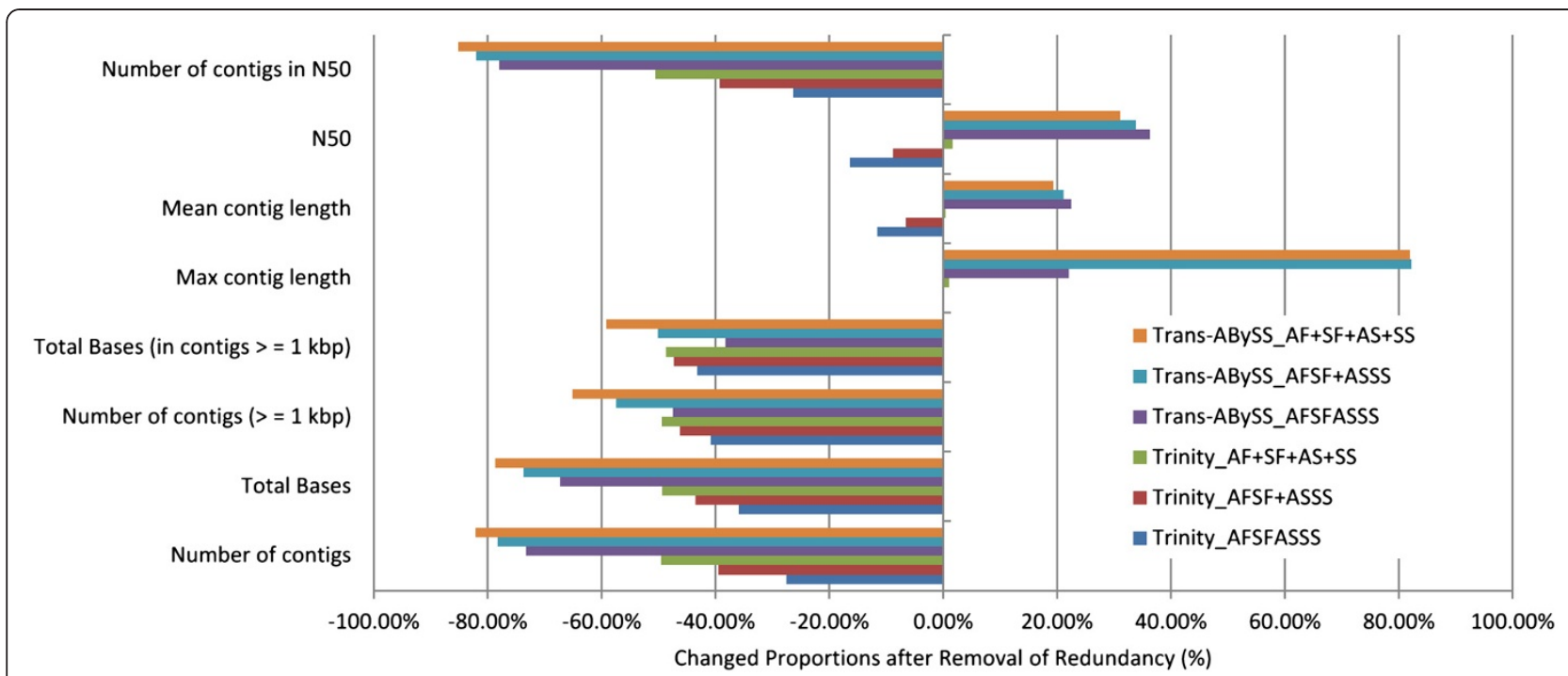

Figure 2 Effects of removal of redundancy. The influence of removal of redundancy on the six assemblies, shown as a changed proportion.

Reads from four samples were mapped to the six assemblies separately. With the exception of Trinity_AFSFASSS, the proportions of reads for the four samples mapped to the other five assemblies were equivalent, indicating that the assemblies did not favor certain samples. As for Trinity_AFSFASSS, the proportion of mapped reads from the SS sample was only $74.98 \%$, and those for AF, AS, and SF were all above $80 \%$, suggesting that Trinity_AFSFASSS was less represented in the SS sample. In the instance of the AF sample, the proportion of reads mapped to Trans-ABySS_AFSFASSS, Trans-

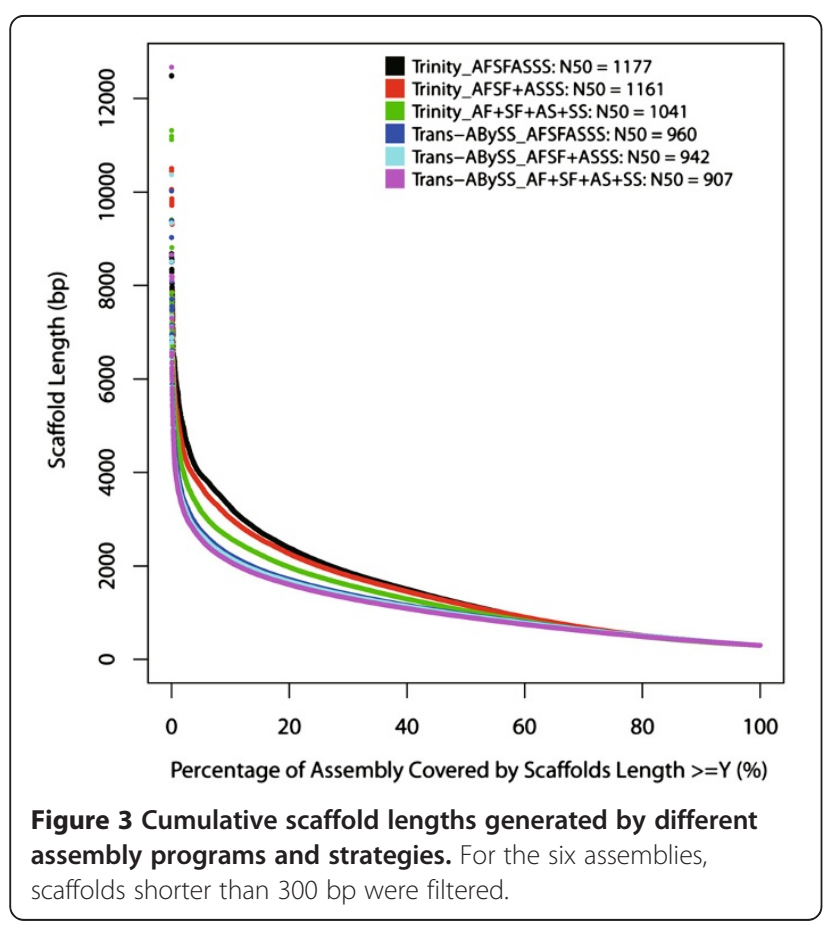

ABySS_AFSF + ASSS, and Trans-ABySS_AF + SF + AS + SS was $93.62 \%, 92.44 \%$, and $91.48 \%$, respectively, suggesting that for Trans-ABySS assemblies, the more assemblies that were merged, the less reads were mapped, which differs from Trinity assemblies. Merging individual Trinity assemblies increased mappable reads.

\section{Assessment of continuity and accuracy}

A full-length common wheat cDNA dataset from TriFLDB was used to evaluate assembly continuity [19]. This dataset contained 6,137 sequences ranging from $131 \mathrm{bp}$ to $8,930 \mathrm{bp}$ in length (N50 of 1,966 bp). Six assemblies were used to BLAT against these full-length transcripts. The proportion of numbers and bases of full-length transcripts covered by each of the six assemblies was calculated and are shown in Table 5. Approximately $90 \%$ of full-length cDNAs were matched by the six assemblies separately, and nearly all matched assembled transcripts covering over $90 \%$ of the fulllength cDNA in length. Trinity outperformed TransABySS in assembly continuity (Table 5), and for both assemblers, merging individual assemblies decreased contig length, particularly for Trans-ABySS.

Another metric of assembly performance is transcript accuracy. To gain a general overview of transcript accuracy, a custom EST library, constructed using the recurrent parent Chinese Spring (CS) containing 51,449 Sanger-sequenced ESTs ranging from $100 \mathrm{bp}$ to $922 \mathrm{bp}$ in length (N50 of $634 \mathrm{bp}$ ) was used (Zhao GY et al., unpublished). With the threshold minimum of $98 \%$ base identity, Trans-ABySS_AFSFASSS covered $81.52 \%$ CS ESTs bases, which was greater than the other five assemblies. On average, Trans-ABySS assemblies were much 


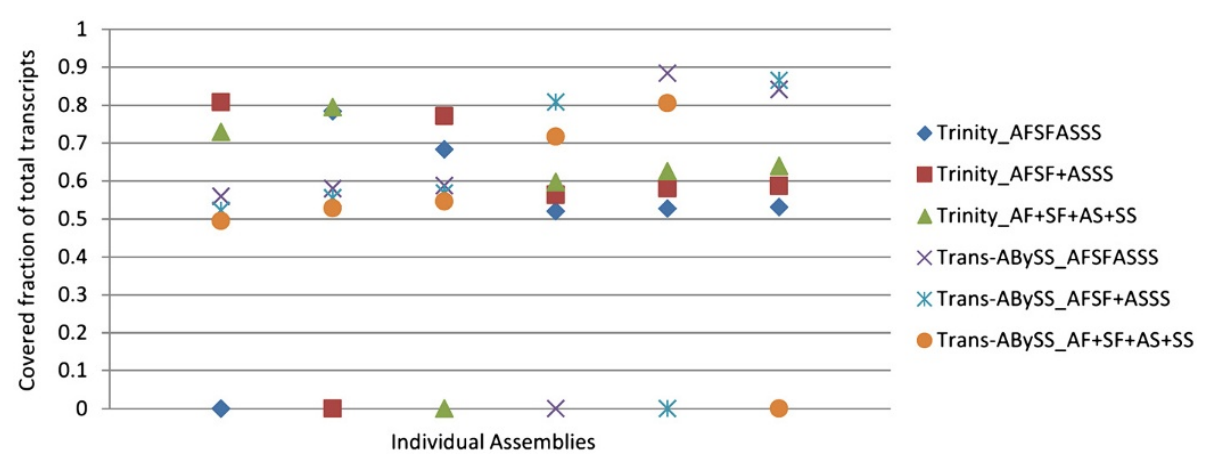

Figure 4 Pairwise comparisons between the six assemblies. Assemblies were compared in a pair-wise fashion using BLAT, and the proportions covered are shown.

more similar to the CS ESTs than those of Trinity (Table 6).

\section{Comparisons with public wheat ESTs}

To compare the six assemblies to other publicly available transcript data, all common wheat ESTs deposited at $\mathrm{dbEST/GenBank}$ were downloaded (1,073,845 ESTs with an N50 of 639 bp, 2012.01.24). Similarity searches against this EST dataset were performed (Table 7). The proportions of public common wheat ESTs bases covered by these six assemblies were all approximately $80 \%$, and the highest one was Trans-ABySS_AFSFASSS. In general, approximately $80 \%$ of EST bases were covered by these six assemblies. However, the proportions of the six assemblies covered by this EST dataset varied, with the lowest being $57.09 \%$ from Trinity_AFSFASSS to the highest being 75.90\% from Trans-ABySS_AF + SF + AS + SS.

\section{Size of wheat transcriptome}

The sizes of the six assemblies varied from 97.9 $\mathrm{Mb}$ to 151.4 Mb. These transcripts were mapped to a draft genomic sequence of Ae. tauschii, which is the diploid progenitor of common wheat (Jia et al., unpublished). We selected only 237,145 scaffolds ranging from 300 bp to $720,471 \mathrm{bp}$ in length from the $\mathrm{D}$ genome sequence to perform the alignments. Assembled transcripts from the six assemblies were aligned to the draft reference sequence using BWASW with default parameters (a mode of BWA) [20], and the numbers of covered bases for the diploid sequence were calculated as shown in Table 8 . On average, approximately two transcripts aligned at a single locus for all six assemblies, whereas the coverage range differed dramatically. Trinity_AFSFASSS covered about $62.1 \mathrm{Mb}$ for this diploid draft genome, which was the most among the six assemblies (Table 8).

Table 4 Statistics of reads mapped to assemblies

\begin{tabular}{|c|c|c|c|c|c|c|}
\hline \multirow[t]{2}{*}{ Proportion of reads } & \multicolumn{3}{|c|}{ Trinity } & \multicolumn{3}{|c|}{ Trans-ABySS } \\
\hline & AFSFASSS & AFSF + ASSS & $A F+S F+A S+S S$ & AFSFASSS & AFSF + ASSS & $A F+S F+A S+S S$ \\
\hline AF aligned & $83.83 \%$ & $87.53 \%$ & $89.17 \%$ & $93.62 \%$ & $92.44 \%$ & $91.48 \%$ \\
\hline aligned more than 3 hits & $4.18 \%$ & $8.12 \%$ & $9.63 \%$ & $2.93 \%$ & $3.89 \%$ & $4.91 \%$ \\
\hline uniquely aligned & $51.85 \%$ & $48.67 \%$ & $48.48 \%$ & $71.74 \%$ & $67.46 \%$ & $63.89 \%$ \\
\hline ASaligned & $82.21 \%$ & $86.50 \%$ & $88.27 \%$ & $92.82 \%$ & $91.85 \%$ & $90.73 \%$ \\
\hline aligned more than 3 hits & $4.23 \%$ & $8.35 \%$ & $10.45 \%$ & $3.00 \%$ & $3.85 \%$ & $4.93 \%$ \\
\hline uniquely aligned & $50.67 \%$ & $48.48 \%$ & $47.70 \%$ & $70.94 \%$ & $66.89 \%$ & $62.74 \%$ \\
\hline SF aligned & $80.06 \%$ & $86.13 \%$ & $88.61 \%$ & $93.55 \%$ & $92.23 \%$ & $91.41 \%$ \\
\hline aligned more than 3 hits & $3.88 \%$ & $8.30 \%$ & $11.46 \%$ & $3.92 \%$ & $4.79 \%$ & $6.08 \%$ \\
\hline uniquely aligned & $50.48 \%$ & $48.73 \%$ & $47.06 \%$ & $71.63 \%$ & $67.09 \%$ & $62.58 \%$ \\
\hline SS aligned & $74.98 \%$ & $86.39 \%$ & $88.47 \%$ & $92.67 \%$ & $91.68 \%$ & $90.81 \%$ \\
\hline aligned more than 3 hits & $3.81 \%$ & $7.83 \%$ & $10.71 \%$ & $3.19 \%$ & $3.98 \%$ & $5.07 \%$ \\
\hline uniquely aligned & $46.73 \%$ & $51.45 \%$ & $49.93 \%$ & $72.35 \%$ & $68.42 \%$ & $64.23 \%$ \\
\hline All aligned & $79.89 \%$ & $86.57 \%$ & $88.52 \%$ & $93.01 \%$ & $91.95 \%$ & $90.98 \%$ \\
\hline aligned more than 3 hits & $4.04 \%$ & $8.15 \%$ & $10.56 \%$ & $3.19 \%$ & $4.04 \%$ & $5.15 \%$ \\
\hline uniquely aligned & $49.60 \%$ & $49.47 \%$ & $48.41 \%$ & $71.61 \%$ & $67.48 \%$ & $63.35 \%$ \\
\hline
\end{tabular}


Table 5 Comparisons between full-length cDNA transcripts and assembled transcripts

\begin{tabular}{|c|c|c|c|c|c|c|}
\hline \multirow[t]{2}{*}{$6,137 \mathrm{fl}-\mathrm{cDNA}$ transcripts } & \multicolumn{3}{|c|}{ Trinity } & \multicolumn{3}{|c|}{ Trans-ABySS } \\
\hline & AFSFASSS & AFSF + ASSS & $A F+S F+A S+S S$ & AFSFASSS & AFSF + ASSS & $A F+S F+A S+S S$ \\
\hline$\%$ of fl-cDNA hit & $92.94 \%$ & $92.83 \%$ & $92.62 \%$ & $91.33 \%$ & $90.39 \%$ & $89.69 \%$ \\
\hline (\% of bases covered) & $82.84 \%$ & $82.64 \%$ & $81.68 \%$ & $79.52 \%$ & $76.71 \%$ & $74.09 \%$ \\
\hline$\%$ of $\mathrm{fl}-\mathrm{cDNA}$ hit with at least $90 \%$ of its length & $92.06 \%$ & $92.05 \%$ & $92.13 \%$ & $90.83 \%$ & $89.73 \%$ & $88.90 \%$ \\
\hline (\% of bases covered) & $82.08 \%$ & $82.02 \%$ & $81.14 \%$ & $79.24 \%$ & $76.40 \%$ & $73.79 \%$ \\
\hline
\end{tabular}

\section{Discussion and conclusions}

In this study, we constructed six wheat transcriptome assemblies using different assemblers and merging strategies on 16.2 Gb PE data. We divided the assembly processes into the following four steps: pre-assembly, merging samples, removal of redundancy, and scaffolding. We recorded the effects of each step in detail. The yielded six assemblies had promising N50 (Table 3), continuity (Table 5), and accuracy (Table 6).

\section{Performance comparisons between Trinity and Trans- ABySS}

Trinity, which uses the single k-mer method, outperformed Trans-ABySS in assembly continuity. The average N50 of the three Trinity assemblies was 16.7\% longer than that of Trans-ABySS (Table 3). All three Trinity assemblies covered more bases of full-length cDNA than their counterpart Trans-ABySS assemblies (Table 5). After the same procedures, Trans-ABySS assemblies, which were produced by the multiple k-mer method, were less redundant (Table 4) and covered a greater proportion of existing Sanger-derived ESTs (Tables 6, 7), representing a good spectrum of expression levels.

Pairwise comparisons of these six assemblies suggested that assemblies produced by the same assembler were more similar to each other. There was a tradeoff between specificity and sensitivity based on the choice of k-mer size. Assemblies with larger k-mers had fewer spurious overlaps but lower coverage. Low-expression genes were assembled more effectively with small k-mer sizes, leading to the assembly of numerous and highly fragmented transcripts, whereas high-expression genes were assembled more effectively with large k-mer sizes, emphasizing contiguity $[12,14]$. For Trinity, we used the default k-mer size of 25, while Trans-ABySS used every odd number starting from 45 to 87 . To test whether the different k-mer sets used by Trinity and Trans-ABySS caused the low level of overlapping regions, we reassembled an assembly with all four samples together using a single $\mathrm{k}$-mer size of 25 , as implemented by ABySS version 1.2.5 [21], named ABySS_AFSFASSS_k25. When ABySS_AFSFASSS_k25 was compared with Trinity_AFSFASSS, $40.9 \%$ of Trinity_AFSFASSS bases were covered, which was lower than the $56.0 \%$ of bases covered by Trans-ABySS_AFSFASSS (Figure 4, Additional file 3: Table S2). This suggested that when using the same k-mer size of 25, ABySS and Trinity still had a large proportion of unique bases. For the initial assemblies, Trans-ABySS produced assemblies that had more contigs that were much larger than those of Trinity (Table 2). For this dataset (mean reads length of about $75 \mathrm{bp}$ ), both Trinity and Trans-ABySS had good performance, and the majority of reads were able to map to the six final assemblies, particularly for the Trans-ABySS_AFSFASSS (Table 4).

\section{Merging assemblies of individual samples}

RNA-Seq analyses often deal with multiple samples. In this study, we compared the performances of different assemblers and merging strategies. The greatest concern for assembling all samples at the initial step is the loss of sample-specific transcripts. With the exception of Trinity_AFSFASSS, the other five assemblies, including Trans-ABySS_AFSFASSS, had equal proportions of the four samples, suggesting that using reads from all samples together may not always introduce bias from overor under-represented samples.

For the single k-mer assembler Trinity, merging assemblies of individual samples increased the number of mappable reads (Table 4) and the proportion of bases covered from full-length cDNA and public ESTs (Tables 6, 7),

Table 6 Comparisons between Chinese Spring cDNA transcripts and assembled transcripts

\begin{tabular}{|c|c|c|c|c|c|c|}
\hline \multirow[t]{2}{*}{ 51,449 Chinese Spring (CS) ESTs } & \multicolumn{3}{|c|}{ Trinity } & \multicolumn{3}{|c|}{ Trans-ABySS } \\
\hline & AFSFASSS & AFSF + ASSS & $A F+S F+A S+S S$ & AFSFASSS & AFSF + ASSS & $A F+S F+A S+S S$ \\
\hline$\%$ of CS ESTs hit & $92.64 \%$ & $93.97 \%$ & $93.60 \%$ & $92.89 \%$ & $91.65 \%$ & $90.63 \%$ \\
\hline (\% of bases covered) & $85.82 \%$ & $87.97 \%$ & $87.91 \%$ & $87.73 \%$ & $85.72 \%$ & $83.99 \%$ \\
\hline$\%$ of CS ESTs hit with at least $98 \%$ of identity & $51.56 \%$ & $65.21 \%$ & $76.07 \%$ & $73.98 \%$ & $71.58 \%$ & $69.19 \%$ \\
\hline (\% of bases covered) & $59.46 \%$ & $65.15 \%$ & $69.54 \%$ & $81.52 \%$ & $79.25 \%$ & $77.08 \%$ \\
\hline
\end{tabular}


Table 7 Comparisons between public ESTs and assembled transcripts

\begin{tabular}{|c|c|c|c|c|c|c|}
\hline & \multicolumn{3}{|c|}{ Trinity } & \multicolumn{3}{|c|}{ Trans-ABySS } \\
\hline & AFSFASSS & AFSF + ASSS & $A F+S F+A S+S S$ & AFSFASSS & AFSF + ASSS & $\mathrm{AF}+\mathrm{SF}+\mathrm{AS}+\mathrm{SS}$ \\
\hline$\%$ of ESTs hit ${ }^{a}$ & $87.83 \%$ & $88.89 \%$ & $88.86 \%$ & $88.64 \%$ & $87.91 \%$ & $87.29 \%$ \\
\hline (\% of bases covered) & $77.76 \%$ & $79.76 \%$ & $80.04 \%$ & $80.49 \%$ & $79.50 \%$ & $78.61 \%$ \\
\hline$\%$ of assembled transcripts hit ${ }^{\mathrm{b}}$ & $59.54 \%$ & $65.43 \%$ & $69.82 \%$ & $78.05 \%$ & $80.58 \%$ & $80.78 \%$ \\
\hline (\% of bases covered) & $57.09 \%$ & $62.59 \%$ & $66.09 \%$ & $70.91 \%$ & $74.24 \%$ & $75.90 \%$ \\
\hline
\end{tabular}

a Proportion of public ESTs matched by assembled transcripts.

${ }^{b}$ Proportion of assembled transcripts matched by public ESTs.

suggesting that the merging strategies broadened the coverage of the assemblies produced by Trinity. Alternatively, merging Trinity assemblies slightly decreased the base coverage of full-length transcripts (Table 5) and N50 (Table 3), suggesting that merging assemblies from individual samples decreased continuity. The main defect was the substantial introduction of redundancy, as indicated by the decreased number of uniquely mapped reads and increased number of repetitively mapped reads (Table 4).

For the multiple k-mer assembler Trans-ABySS, merging assemblies from individual samples had the following shortcomings: shorter N50 (Table 3), fewer number of mappable reads, reduced proportions of bases covered from full-length cDNA and public ESTs, and slightly increased redundancy (Table 4).

\section{Removal of redundancy}

A large assembly including transcripts across a wide range of expression levels but containing many redundant contigs/scaffolds could be worse than a small assembly only contain unique bases. Redundancy can pose difficulties in the downstream analysis.

After merging assemblies from individual samples, the overall contig numbers from these six assemblies were still very high (ranging from 227,879 to 720,131 ), even when considering the various transcript isoforms (Table 3, Additional file 2: Table S1). The reason might be sequencing errors and potential sequence variations among individual plants. Thus, we implemented a clustering step to remove redundancy. A major concern of removing redundancy is the mis-assembly of highly similar transcripts. Although there is no straightforward way to evaluate the performance of this step due to the lack of well-defined genomic sequences, we used two datasets to assess the parameters we used: one with 51 WRKY transcription factors detected from common wheat UniGenes, and the other with 23 groups (a total 69 sequences) of orthologous genes from A, B, and D subgenomes of hexaploid wheat, representing conserved gene families and highly similar but distinct sequences, respectively (Additional file 4: Table S3). With the thresholds of a minimum length of $50 \mathrm{bp}$ and minimum identity of $99 \%$, none of these sequences were assembled together suggesting the parameters we used in removing redundancy is proper.

After removing duplications using this strict standard, for the Trinity_AFSFASSS that was assembled without merging individual assemblies, $27.52 \%$ of its contig number and $35.87 \%$ of its total length had been reduced. To compare the effect of deduplication, we reproduced an assembly (Trinity_AFSFASSS_2 ${ }^{\text {nd }}$ ) that used every procedure used for assembling the Trinity_AFSFASSS without the step that removes redundancy. Trinity_AFSFASSS_2 $2^{\text {nd }}$ had an N50 of 1,385 bp contained 225,280 scaffolds ranging from $300 \mathrm{bp}$ to $12,488 \mathrm{bp}$ in length. Trinity_AFSFASSS_2 ${ }^{\text {nd }}$, with a total size of $217,468,671$ bp, was mapped to the draft diploid Ae. tauschii sequence using the same parameters used for Trinity_AFSFASSS. Trinity_AFSFASSS_2 ${ }^{\text {nd }}$ covered about $63.0 \mathrm{Mb}$, which was equivalent to that of Trinity_AFSFASSS, suggesting that the removing the redundancy step that we used may not greatly reduce unique transcripts and was appropriate to use (Table 8). To further compare the effects of removing redundancy, reads from the four samples were mapped to Trinity_AFSFASSS_2 ${ }^{\text {nd }}$. Comparing to Trinity_AFSFASSS, the total number of mappable reads slightly decreased by $1.05 \%$. Moreover, the proportion of reads mapped to more

Table 8 Statistic of transcripts aligned to the draft diploid Ae. tauschii genome

\begin{tabular}{|c|c|c|c|c|c|c|c|c|}
\hline & \multicolumn{3}{|c|}{ Trinity } & \multicolumn{3}{|c|}{ Trans-ABySS } & \multirow[t]{2}{*}{ Public ESTs } & \multirow[t]{2}{*}{ Trinity_AFSFASSS_2 $2^{\text {nd }}$} \\
\hline & AFSFASSS & AFSF + ASSS & $A F+S F+A S+S S$ & AFSFASSS & AFSF + ASSS & $A F+S F+A S+S S$ & & \\
\hline Mean coverage & 1.9 & 2.2 & 2.3 & 2 & 2.1 & 2.1 & 11.0 & 2.9 \\
\hline Coverage range & $1-279$ & $1-339$ & $1-254$ & $1-26$ & $1-24$ & $1-24$ & $1-10,122$ & $1-658$ \\
\hline Bases covered & $62,103,048$ & $58,923,609$ & $56,105,411$ & $51,499,767$ & $45,678,989$ & $41,571,199$ & $46,506,263$ & $63,042,809$ \\
\hline
\end{tabular}

Assembled transcripts were aligned to the draft genomic sequence. For the covered regions of the reference by assembled transcripts, mean coverage indicated how many transcripts covered on average for a certain region. 
than three locations increased from $4.04 \%$ to $12.95 \%$, and the uniquely mapped reads were reduced from $49.60 \%$ to 31.38\% (Additional file 5: Table S4). It is probably difficult for this assembly to generate accurate overall downstream RNA-Seq analyses, suggesting that proper removal of redundancy may be crucial for polyploidy plant transcriptome assembly.

\section{Using assembled ESTs or de novo assemblies as references}

In general, only uniquely mapped reads can be used in common downstream RNA-Seq analyses, including SNP discovery and the identification of genes that are differentially expressed between samples. This was the reason we used mapped reads, especially the number of uniquely mapped reads as a measurement of assembly performances. Considering the large amount of common wheat cDNA that already exists in the public domain, we mapped all reads to the following two most commonly used assembled EST datasets: wheat gene index (TAGI) and wheat PUT assembly (TAPUT) [22,23] (Additional file 5: Table S4). The proportions of mappable reads, repetitively mapped reads, and uniquely mapped reads were $83.36 \%$ and $81.34 \%, 25.00 \%$ and $15.10 \%, 43.77 \%$ and $51.04 \%$ of TAGI and TAPUT, respectively. When compared with the results of de novo assemblies, a more accurate overview of the wheat transcriptome can be obtained using de novo assemblies as references, particularly the Trans-ABySS_AFSFASSS (Table 4).

The GC content of the six de novo assemblies was about $48 \%$ (Table 3), and those of 1,073,845 common wheat ESTs, TAGI and TAPUT were 52.33\%, 52.10\% and $51.68 \%$, respectively. The lower level of GC content for these assemblies may be due to filtered reads, which were already GC content-less-presented (Table 1).

\section{Construction of wheat transcriptome with short reads}

Due to the lack of a well-annotated genomic sequence, we could not assembly the wheat transcriptome using a mapping-first strategy. To assess the performance of assembling the wheat transcriptome using short reads, we used the existing public wheat ESTs, a custom Chinese Spring cDNA library and a draft genome sequence of Ae. tauschii, the wheat $\mathrm{D}$ genome progenitor.

By mapping assembled transcripts back to the draft Ae. tauschii sequence, the number of bases covered by each of these assemblies was used to compare the total number of bases constructed from each assembly. Combining the length of N50 for these assemblies from both assemblers, the more reads that were used at the initial assembling step, the more unique bases that were constructed in the final assemblies. Considering that we had used the maximum of $16.2 \mathrm{~Gb}$ reads at the pre- assembling step, which was already more than 100 times larger than the size of the final assemblies, further improvements in assembly may need even more reads or longer reads. The use of $16.2 \mathrm{~Gb}$ data has already provided promising results. Nearly all assembled transcripts, which were matched by full-length cDNA using BLAT, covered $90 \%$ of full length cDNA in length (Table 5), suggesting that the length of transcripts assembled by Illumina reads was acceptable.

On average, more than one transcript was mapped to the same locations of the draft Ae. tauschii sequence for all six assemblies (Table 8), which was expected, because the reference is diploid while the transcriptome is from hexaploid wheat.

To compare the assemblies with existing wheat resources, all of the currently available wheat ESTs were aligned to the same Ae. tauschii sequence. A total of $1,073,845$ common wheat ESTs deposited in GenBank covered $46.5 \mathrm{Mb}$ of reference sequences, which was less than that of four of the six assemblies (Table 8). Considering the direct comparison results between these assemblies and public ESTs (Table 7), this study also provided considerable new wheat transcript resources to the research community.

\section{Methods}

\section{Plant materials}

Wheat tissues were collected from Taigu/CS nearisogenic lines (NILs), which were developed by backcrossing the "sterile" donor cultivar Taigu with the recurrent parent Chinese Spring (CS). Taigu carries the dominant sterile gene $m s 2$. Mixed anther samples with lengths of $0.5-2.5 \mathrm{~mm}$ were collected from fertile and sterile plants and were designated as AF and AS, respectively. Mixed young spike samples with lengths of $3.0-4.5 \mathrm{~cm}$ were collected from fertile and sterile plants and were designated as SF and SS, respectively.

\section{RNA extraction, library construction and Illumina sequencing}

Total RNA was extracted using TRIzol reagent (Life Technologies, Grand Island, NY). The quality and quantity of RNA were examined using an Agilent 2100 Bioanalyzer (Agilent Technologies, Santa Clara, CA) before the following procedures: enrichment of mRNA by Oligo(dT), fragmentation, cDNA synthesis by random hexamer primers, size selection (200 bp) and PCR amplification, which was performed by BGI-Senzhen as described previously [9]. RNA sequencing was performed on a HiSeq 2000 (Illumina, San Diego, CA).

\section{Raw reads filtering}

Pair-end raw reads were trimmed with the BWA trimming mode at a threshold of Q13 $(P=0.05)$ as 
implemented by SolexaQA version 1.10 [24]. Lowquality 3' ends of each read were filtered. Reads that were less than $25 \mathrm{bp}$ in length or were singletons were discarded.

\section{Pre-assembly of short reads}

De novo assembly was performed using both Trinity and Trans-ABySS. Trinity release 20110519 was employed with the ALLPATHSLG error correction, and the paired fragment length was set to $200 \mathrm{bp}$ [11]. Individual k-mer assemblies were carried out by ABySS version 1.2.5 with the scaffolding option off and contig end erosion off [21]. Trans-ABySS version 1.2.0 was used to merge the individual $\mathrm{k}$-mer assemblies with default parameters [12].

\section{Merging, deduplication and scaffolding}

Assemblies of individual samples were merged with both strands information using the accurate mode of cd-hitest version 4.5.4 with the sequence identity threshold at $100 \%$ and a word size of 8 [15]. GICL (release date 2010-07-22) was used to remove redundancy [16]. Contigs overlapped with at least $50 \mathrm{bp}$ with a minimum identity of $99 \%$ were collapsed into single contigs, and the maximum length of unmatched overhangs was set to 100 bp. Triticum aestivum UniGene Build 60 was downloaded from NCBI. One clone-derived sequence selected from each UniGene cluster was used to perform the domain detection. WRKY HMM was downloaded from Pfam [25]. Genewisedb module from wise2 package (version 2.4.1) was used at the threshold of 1E-8 [26]. Scaffolding pre-assembled contigs was performed using SSPACE version 1.1 [17]. The command used for SSPACE was "-x 0 -m 50 -o 20 -k 5 -n 15". The TransABySS_AFSFASSS assembly with the highest mappable reads among the six assemblies was deposited in GenBank/TSA under the accession Nos. JV812965-JV999999 and JW000001-JW036047. The other five assemblies are available upon request.

\section{Short-read alignment}

Short reads were aligned to references using BWA version 0.6.1 [27] with the default parameters, expect the seeding option was turned off to ensure that mismatches were allowed to be randomly distributed along the reads. Manipulating alignment results involved the use of SAMtools version 0.1.18 [28].

\section{Assembled transcripts alignment}

The BWASW mode in BWA was used to align assembled transcripts into the draft diploid reference with default parameters [20]. Comparisons of transcripts were performed using BLAT version 34x12 with default parameters, and the alignment results were filtered with various demands [18]. The assembled two EST datasets used in analyses were TAGI release 12.0 and wheat PUT assembly version $163 \mathrm{~b}[16,23]$.

\section{Additional files}

Additional file 1: Figure S1. Effects of merging assemblies of different samples and scaffolding (shown as changed proportions).

Additional file 2: Table S1. Detailed statistics for each assembly step. Additional file 3: Table S2. Detailed pairwise comparisons between the six assemblies.

Additional file 4: Table S3. Sequences from A, B and D subgenome used in the assessment of parameters used in the step of removing redundancy.

Additional file 5: Table S4. Statistics of reads mapping to the assembled EST datasets (TAGl and TAPUT).

Abbreviations

PE: Pair end; CS: Chinese spring; fl-cDNA: Full-length cDNA.

\section{Competing interests}

The authors declare that they have no competing interests.

\section{Authors' contributions}

Conceived and designed the experiments: JD CX JJ XK. Performed the experiments: CX. Performed the bioinformatics analysis: JD. Contributed reagents/materials/analysis tools: GZ. Wrote the paper: JD JJ XK. All authors read and approved the final manuscript.

\section{Acknowledgements}

This work was supported by grants from National Basic Research Program of China (2010CB125902) and National Transgenic Research Project (2009ZX08009-110B).

\section{Author details}

${ }^{1}$ Key Laboratory of Crop Gene Resources and Germplasm Enhancement, Ministry of Agriculture, National Key Facility for Crop Gene Resources and Genetic Improvement, Institute of Crop Science, Chinese Academy of Agricultural Sciences, No. 12 South Street, Zhongguancun, Beijing 100081, People's Republic of China. ${ }^{2}$ College of Biology Sciences, China Agricultural University, No. 2 Yuanmingyuan West Road, Haidian District, Beijing 100094, People's Republic of China.

Received: 27 March 2012 Accepted: 3 August 2012

Published: 14 August 2012

\section{References}

1. Feng C, Chen M, Xu CJ, Bai L, Yin XR, Li X, Allan AC, Ferguson IB, Chen KS: Transcriptomic analysis of Chinese bayberry (Myrica rubra) fruit development and ripening using RNA-Seq. BMC Genomics 2012, 13:19.

2. Zhang G, Guo G, Hu X, Zhang Y, Li Q, Li R, Zhuang R, Lu Z, He Z, Fang X, et al: Deep RNA sequencing at single base-pair resolution reveals high complexity of the rice transcriptome. Genome Res 2010, 20(5):646-654

3. Ness RW, Siol M, Barrett SC: De novo sequence assembly and characterization of the floral transcriptome in cross- and self-fertilizing plants. BMC Genomics 2011, 12:298.

4. Bundock PC, Eliott FG, Ablett G, Benson AD, Casu RE, Aitken KS, Henry RJ: Targeted single nucleotide polymorphism (SNP) discovery in a highly polyploid plant species using 454 sequencing. Plant Biotechnol J 2009, 7(4):347-354.

5. Trick M, Adamski NM, Mugford SG, Jiang CC, Febrer M, Uauy C: Combining SNP discovery from next-generation sequencing data with bulked segregant analysis (BSA) to fine-map genes in polyploid wheat. BMC Plant Biol 2012, 12:14. 
6. Pellny TK, Lovegrove A, Freeman J, Tosi P, Love CG, Knox JP, Shewry PR, Mitchell RA: Cell walls of developing wheat starchy endosperm: comparison of composition and RNA-Seq transcriptome. Plant Physiol 2012, 158(2):612-627.

7. Pont C, Murat F, Confolent C, Balzergue S, Salse J: RNA-seq in grain unveils fate of neo- and paleopolyploidization events in bread wheat (Triticum aestivum L.). Genome Biol 2011, 12(12):R119.

8. Li A, Zhang R, Pan L, Tang L, Zhao G, Zhu M, Chu J, Sun X, Wei B, Zhang X, et al: Transcriptome analysis of $\mathrm{H}_{2} \mathrm{O}_{2}$-treated wheat seedlings reveals a $\mathrm{H}_{2} \mathrm{O}_{2}$-responsive fatty acid desaturase gene participating in powdery mildew resistance. PLoS One 2011, 6(12):e28810.

9. Zhang J, Liang S, Duan J, Wang J, Chen S, Cheng Z, Zhang Q, Liang X, Li Y: De novo assembly and characterisation of the transcriptome during seed development, and generation of genic-SSR markers in peanut (Arachis hypogaea L.). BMC Genomics 2012, 13(1):90.

10. Schulz MH, Zerbino DR, Vingron M, Birney E: Oases: robust de novo RNAseq assembly across the dynamic range of expression levels. Bioinformatics 2012, 28(8):1086-1092.

11. Grabherr MG, Haas BJ, Yassour M, Levin JZ, Thompson DA, Amit I, Adiconis X, Fan L, Raychowdhury R, Zeng Q, et al: Full-length transcriptome assembly from RNA-Seq data without a reference genome. Nat Biotechnol 2011, 29(7):644-652.

12. Robertson G, Schein J, Chiu R, Corbett R, Field M, Jackman SD, Mungall K, Lee S, Okada HM, Qian JQ, et al: De novo assembly and analysis of RNAseq data. Nat Methods 2010, 7(11):909-912.

13. Cantu D, Pearce SP, Distelfeld A, Christiansen MW, Uauy C, Akhunov E, Fahima T, Dubcovsky J: Effect of the down-regulation of the high Grain Protein Content (GPC) genes on the wheat transcriptome during monocarpic senescence. BMC Genomics 2011, 12:492.

14. Surget-Groba Y, Montoya-Burgos J: Optimization of de novo transcriptome assembly from next-generation sequencing data. Genome Res 2010, 20(10):1432-1440.

15. Li W, Godzik A: Cd-hit: a fast program for clustering and comparing large sets of protein or nucleotide sequences. Bioinformatics 2006, 22(13):1658-1659.

16. Pertea G, Huang X, Liang F, Antonescu V, Sultana R, Karamycheva S, Lee Y, White J, Cheung F, Parvizi B, et al: TIGR Gene Indices clustering tools (TGICL): a software system for fast clustering of large EST datasets. Bioinformatics 2003, 19(5):651-652.

17. Boetzer M, Henkel CV, Jansen HJ, Butler D, Pirovano W: Scaffolding preassembled contigs using SSPACE. Bioinformatics 2011, 27(4):578-579.

18. Kent WJ: BLAT-the BLAST-like alignment tool. Genome Res 2002, 12(4):656-664.

19. Mochida K, Yoshida T, Sakurai T, Ogihara Y, Shinozaki K: TriFLDB: a database of clustered full-length coding sequences from Triticeae with applications to comparative grass genomics. Plant Physiol 2009, 150(3):1135-1146.

20. Li H, Durbin R: Fast and accurate long-read alignment with Burrows-Wheeler transform. Bioinformatics 2010, 26(5):589-595

21. Birol I, Jackman SD, Nielsen CB, Qian JQ, Varhol R, Stazyk G, Morin RD, Zhao $Y$, Hirst M, Schein JE, et al: De novo transcriptome assembly with ABySS. Bioinformatics 2009, 25(21):2872-2877.

22. Childs KL, Hamilton JP, Zhu W, Ly E, Cheung F, Wu H, Rabinowicz PD, Town CD, Buell CR, Chan AP: The TIGR Plant Transcript Assemblies database. Nucleic Acids Res 2007, 35(Database issue):D846-D851.

23. Duvick J, Fu A, Muppirala U, Sabharwal M, Wilkerson MD, Lawrence CJ, Lushbough C, Brendel V: PlantGDB: a resource for comparative plant genomics. Nucleic Acids Res 2008, 36(Database issue):D959-D965.

24. Cox MP, Peterson DA, Biggs PJ: SolexaQA: At-a-glance quality assessment of Illumina second-generation sequencing data. BMC Bioinformatics 2010, 11:485.

25. Punta M, Coggill PC, Eberhardt RY, Mistry J, Tate J, Boursnell C, Pang N, Forslund K, Ceric G, Clements J, et al: The Pfam protein families database. Nucleic Acids Res 2012, 40(Database issue):D290-301.

26. Birney E, Clamp M, Durbin R: Genewise and genomewise. Genome Res 2004, 14(5):988-995.
27. Li H, Durbin R: Fast and accurate short read alignment with Burrows-Wheeler transform. Bioinformatics 2009, 25(14):1754-1760.

28. Li H, Handsaker B, Wysoker A, Fennell T, Ruan J, Homer N, Marth G, Abecasis G, Durbin R, 1000 Genome Project Data Processing Subgroup: the sequence alignment/map format and SAMtools. Bioinformatics 2009, 25 (16):2078-2079.

doi:10.1186/1471-2164-13-392

Cite this article as: Duan et al: Optimizing de novo common wheat transcriptome assembly using short-read RNA-Seq data. BMC Genomics 2012 13:392.

\section{Submit your next manuscript to BioMed Central and take full advantage of:}

- Convenient online submission

- Thorough peer review

- No space constraints or color figure charges

- Immediate publication on acceptance

- Inclusion in PubMed, CAS, Scopus and Google Scholar

- Research which is freely available for redistribution

Submit your manuscript at www.biomedcentral.com/submit
C) BioMed Central 